\title{
Transcranial Doppler ultrasonography predicts cardiovascular events after TIA
}

\author{
Katrin Holzer ${ }^{1}$, Suwad Sadikovic ${ }^{* 1}$, Lorena Esposito ${ }^{1}$, Angelina Bockelbrink ${ }^{2}$, \\ Dirk Sander ${ }^{3}$, Bernhard Hemmer ${ }^{1}$ and Holger Poppert ${ }^{1}$
}

\author{
Address: ${ }^{1}$ Department of Neurology, Klinikum rechts der Isar, Technische Universität, Munich, Germany, ${ }^{2}$ Institute for Social Medicine, \\ Epidemiology, and Health Economics, Charité University Medical Centre, Berlin, Germany and ${ }^{3}$ Department of Neurology, Medical Park Hospital, \\ Bischofswiesen, Germany \\ Email: Katrin Holzer - katrin.bruckmaier@gmx.de; Suwad Sadikovic* - sadikovic@lrz.tum.de; Lorena Esposito - lorenaesposito@web.de; \\ Angelina Bockelbrink - angelina.bockelbrink@charite.de; Dirk Sander - d.sander@mac.com; Bernhard Hemmer - hemmer@lrz.tu-muenchen.de; \\ Holger Poppert - poppert@neurovasc.de \\ * Corresponding author
}

Published: 30 July 2009

BMC Medical Imaging 2009, 9:13 doi:10.1 186/1471-2342-9-13
Received: 30 January 2009

Accepted: 30 July 2009

This article is available from: http://www.biomedcentral.com/I47/-2342/9//3

(C) 2009 Holzer et al; licensee BioMed Central Ltd.

This is an Open Access article distributed under the terms of the Creative Commons Attribution License (http://creativecommons.org/licenses/by/2.0), which permits unrestricted use, distribution, and reproduction in any medium, provided the original work is properly cited.

\begin{abstract}
Background: Transient ischemic attack (TIA) patients are at high vascular risk. We assessed the value of extracranial $(E C D)$ and transcranial $(T C D)$ Doppler and duplex ultrasonography to predict clinical outcome after TIA.

Methods: 176 consecutive TIA patients admitted to the Stroke Unit were recruited in the study. All patients received diffusion-weighted imaging, standardized ECD and TCD. At a median followup of 27 months, new vascular events were recorded.

Results: 22 (13.8\%) patients experienced an ischemic stroke or TIA, 5 (3.1\%) a myocardial infarction or acute coronary syndrome, and $5(3.1 \%)$ underwent arterial revascularization. ECD revealed extracranial $\geq 50 \%$ stenosis or occlusions in $34(19.3 \%)$ patients, TCD showed intracranial stenosis in $15(9.2 \%)$ and collateral flow patterns due to extracranial stenosis in 5 (3.1\%) cases. Multivariate analysis identified these abnormal ECD and TCD findings as predictors of new cerebral ischemic events (ECD: hazard ratio (HR) 4.30, 95\% confidence interval $(\mathrm{Cl}) \mathrm{I} .75$ to $10.57, \mathrm{P}=0.01$; TCD: HR 4.73, $95 \% \mathrm{Cl} 1.86$ to $12.04, \mathrm{P}=0.0 \mathrm{I})$. Abnormal TCD findings were also predictive of cardiovascular ischemic events (HR I8.5I, 95\% Cl 3.49 to $98.24, \mathrm{P}=0.00 \mathrm{I}$ ).
\end{abstract}

Conclusion: TIA patients with abnormal TCD findings are at high risk to develop further cerebral and cardiovascular ischemic events.

\section{Background}

After a transient ischemic attack (TIA), patients are at high risk to develop further vascular events. The risk of stroke within the first 90 days after TIA is $4 \%$ to $20 \%$, with half of the events occurring within the first 2 days [1-6]. Con- sequently, the early risk of stroke after TIA is comparable to, or even higher than, the short-term risk of myocardial infarction (MI) and major cardiovascular complications in patients presenting with chest pain [7]. Several clinical characteristics such as advanced age $[2,8-10]$, diabetes 
mellitus $[2,9]$, hypertension $[9,10]$, weakness $[2,9,10]$, speech impairment $[2,9,10]$, prolonged symptom duration $[2,8-10]$, evidence of acute ischemia on brain imaging $[3,6,11,12]$, extracranial or intracranial large-artery occlusive disease [3,6,13-15], and cardioembolism [14], have been reported to be independently associated with a higher incidence of early subsequent stroke after TIA. Recently a new scoring system for evaluating short-term stroke risk after TIA ( $\mathrm{ABCD}^{2}$ score) based on 5 clinical factors has been validated $[9,16,17]$. The predictive power of the $\mathrm{ABCD}^{2}$ model seems to be partially explained by identification of those patients likely to have experienced a true TIA [18].

Whereas the risk of stroke is highest in the first year after TIA, ranging from $7 \%$ to $21 \%[1,5,8,19,20]$, and afterwards declines to an annual rate of $2 \%$ to $6 \%$ over the first 4 to 5 years [19-21], the annual risk of coronary events after TIA remains stable at about $2 \%$ to $3 \%$ for several years $[19,20]$. On long-term follow-up, cardiovascular disease becomes the major cause of death after TIA [22]. The 10 -year risk of vascular events in TIA patients is reported to be $36 \%$ and the 10 -year risk of death $34 \%$ respectively [23]. Several studies have already demonstrated a high prevalence of asymptomatic coronary artery disease (CAD) in patients with TIA and mild ischemic stroke (IS), ranging from $28 \%$ to $41 \%$ [24-26]. Routine screening tests for CAD in all patients with cerebrovascular disease may not be cost-effective, however. Healthcare professionals currently are encouraged to optimize coronary risk evaluation in patients with TIA and IS based on the individual cardiovascular risk profile and the prevalence of carotid artery disease [27].

In this study, we aimed to assess the value of extracranial (ECD) and transcranial (TCD) Doppler and duplex ultrasonography to predict the occurrence of cerebrovascular and cardiovascular events after TIA.

\section{Methods}

We identified 262 patients with possible cerebral TIA, who had been consecutively admitted to the Stroke Unit of the Department of Neurology, Technical University Munich, within the first 72 hours after symptom onset between May 2000 and July 2004. Diagnosis was made by the attending neurologist before patient selection. TIA was defined as an acute transient focal neurological deficit caused by vascular disease, which completely reversed within 24 hours [28]. Patients with amaurosis fugax were not included in the study, as data suggest pathogenic and prognostic differences between transient eye and brain ischemic syndromes $[29,30]$.

To be eligible, patients had to undergo cerebral magnetic resonance imaging (MRI) including diffusion-weighted imaging (DWI) sequences within 5 days after onset of symptoms, which was the case in 225 patients. 49 patients were excluded for the following reasons: competing differential diagnosis as assessed by the attending neurologist, 41 cases (migraine, 8 cases; epilepsy, 7 cases; functional disorder, 5 cases; peripheral dizziness, 4 cases; syncope, 4 cases; hypertensive crisis, 4 patients; others, 9 cases); malignancy requiring active treatment, 7 cases; concomitant participation in a pharmaceutical trial; 1 case. Informed consent was obtained of every patient.

Routine admission examinations involved evaluation of medical history, physical examinations, blood analysis including lipid and glucose metabolism, resting and 24hour electrocardiogram, 24-hour blood pressure measurement, transthoracic echocardiography, ECD, TCD, and cerebral MRI including DWI sequences. Symptom duration was systematically documented.

The following baseline clinical data were collected: age, sex, symptom duration, presence of classic vascular risk factors, and medical history of CAD, cardiac failure, and peripheral artery disease (PAD) (Additional file 1). Hypertension was defined as systolic blood pressure $\geq 140$ $\mathrm{mmHg}$, diastolic blood pressure $\geq 90 \mathrm{mmHg}$, or current use of antihypertensive medication; diabetes mellitus as fasting blood glucose $\geq 126 \mathrm{mg} / \mathrm{dL}$ or current use of antidiabetic agents; and hypercholesterolemia as total cholesterol $\geq 240 \mathrm{mg} / \mathrm{dL}$ or current use of lipid-lowering medication. Nicotine abuse was defined as current or former regular smoking. Atrial fibrillation was defined as history of electrocardiographically documented intermittent or persistent atrial fibrillation. TIA was allocated to the carotid or vertebrobasilar territory by an experienced neurologist based on clinical symptoms and MRI.

ECD and TCD were performed within a maximum of 3 days after admission using multi-range doppler (DWL Multi-Dop; Compumedics Germany $\mathrm{GmbH}$ ) and duplex ultrasound devices (Siemens Sonoline Elegra; Siemens $A G)$.

ECD findings were classified as follows: normal (1), if there was no evidence of plaques both in the cervical internal carotid (cICA) and cervical vertebral arteries (cVA); atherosclerosis without stenosis (2), if cICA or cVA showed at least one plaque with $<50 \%$ stenosis of the corresponding vessel; stenosis (3), if CICA or CVA showed at least one $\geq 50 \%$ stenosis or an occlusion. ECD classification did not distinguish between symptomatic and asymptomatic vessel disease.

TCD findings were classified as follows: normal (1), if TCD detected no pathological findings or only minor side-to-side differences of the distal internal carotid 
(dICA), middle cerebral (MCA), posterior cerebral (PCA), or intracranial vertebrobasilar (VBA) artery; reactive collateral flow patterns (2), if TCD demonstrated collateral blood flow through the circle of Willis secondary to extracranial lesions; stenosis (3), if dICA, MCA, PCA, or VBA showed at least one intracranial stenosis or an occlusion. TCD diagnosis of intracranial stenosis was defined by increased peak flow velocities $(\geq 155 \mathrm{~cm} / \mathrm{s}$ for dICA and $\mathrm{MCA} ; \geq 100 \mathrm{~cm} / \mathrm{s}$ for PCA and VBA) with side-to-side differences $>20 \%$ and disturbed flow patterns [31,32]. PCA signal was identified by posterior angulation of the probe during transtemporal insonation using an insonation depth of 60 to $70 \mathrm{~mm}$. For identification of VBA we applied an insonation depth of $6079 \mathrm{~mm}$ (intracranial vertebral arteries) and $80110 \mathrm{~mm}$ (basilar artery) during suboccipital insonation. TCD classification did not distinguish between symptomatic and asymptomatic vessel disease.

Cerebral MRI was performed within a maximum of 5 days after symptom onset in all patients. No patient developed a follow-up cerebrovascular event before MRI.

All MRI scans were obtained using a 1.5-Tesla scanner (Magnetom Symphony; Siemens AG). Imaging protocol included axial T1-weighted (TR/TE 654/14 ms), T2weighted (TR/TE 3305/132 ms), and DWI sequences (TR/ TE $4006 / 83 \mathrm{~ms}$, slice thickness 4 to $6 \mathrm{~mm}$, interslice gap $1.5 \mathrm{~mm}$, pixel matrix $128 \times 128$, field of view $220 \times 220$ $\mathrm{mm}$, pixel size $1.72 \times 1.72 \mathrm{~mm}$, gradient strength $30 \mathrm{mT} /$ $\mathrm{m}$, b-values $=0,500,1000 \mathrm{~s} / \mathrm{mm}^{2}$ ), and in doubtful cases additionally a sagittal or coronal DWI sequence. ADC maps were constructed by linear least-squares fit on a pixel-by-pixel basis after averaging the direction-dependent DWI values.

DWI scans were considered positive for ischemia if both a hyperintensity on the isotropic $b=1000$ scan and a corresponding hypointensity on the ADC map were detectable.

At a median follow-up of 27 months (minimum 4 months, maximum 64 months) all 176 patients were contacted via telephone or mail by an experienced neurologist blinded to the patients' ultrasonographic findings. A semi-structered interview was used to assess new cerebral ischemic or other vascular events. If the interview provided insufficient data or any indication of follow-up events, data were completed by contacting relatives, attending physicians and/or hospitals. Our main points of interest were cerebral ischemic events (ischemic stroke or TIA), cardiovascular ischemic events (MI or acute coronary syndrome (ACS), surgical or endovascular revascularization procedures in CAD or PAD), and death of vascular or unknown cause. Other vascular events and death of nonvascular cause also were documented. If a patient reported symptoms possibly consistent with a fol- low-up event but did not seek medical aid or had competing differential diagnoses as reported by the attending physician, this information was documented but not considered as an outcome event.

All analyses were performed with the SPSS statistical package version 15.0. Univariate Cox regression analysis was used to detect variables associated with the occurrence of endpoints. Cox proportional hazards multivariate analysis adjusted for age and sex was applied to identify independent predictors of cerebral ischemic events, cardiovascular ischemic events, and the combined endpoint of cerebral ischemic events, cardiac ischemic events, and death of vascular or unknown cause. $P<0.05$ was considered as significant. Percentage values are relative to the patent subset with complete data record.

The research protocol was approved by the local ethical committee.

\section{Results}

A total of 176 Caucasian TIA patients were included in the study. Table 1 shows the baseline characteristics of the study population. Medical history revealed former IS, TIA, or amaurosis fugax in $40(23.1 \%)$ patients. $9(5.1 \%)$ patients reported a TIA during the last month before admission.

Mean symptom duration was $4.7 \pm 7.1$ hours, with 65 $(37.6 \%)$ patients having symptoms lasting $<1$ hour. TIA was allocated to the carotid territory in 125 (71.0\%) patients and to the vertebrobasilar territory in $42(23.9 \%)$ patients; 9 further cases $(5.1 \%)$ could not be classified on the basis of symptoms and MRI. DWI showed signal intensity changes suggestive of cerebral ischemia in 49 $(28.3 \%)$ patients.

Table I: Baseline characteristics of study population $(n=176)$

\begin{tabular}{|c|c|}
\hline Age $(y)^{*}$ & $63.3 \pm 14,5$ \\
\hline Sex, female (n) & $67(38.1 \%)$ \\
\hline Hypertension (n) & $127(72.2 \%)$ \\
\hline Diabetes mellitus ( $\mathrm{n}$ ) & $28(15.9 \%)$ \\
\hline Hypercholesterolemia (n) & $84(48.6 \%)$ \\
\hline Body mass index* & $25.8 \pm 3.9$ \\
\hline Nicotine abuse (n) & $80(45.5 \%)$ \\
\hline Atrial fibrillation $(n)$ & $24(13.6 \%)$ \\
\hline Coronary artery disease $(n)$ & 35 (19.9\%) \\
\hline Cardiac failure $(n)$ & $1 \mathrm{I}(6.4 \%)$ \\
\hline Peripheral artery disease $(n)$ & $13(7.4 \%)$ \\
\hline DWI abnormality $(n)$ & $49(28.3 \%)$ \\
\hline Duration $(\mathrm{h})^{*}$ & $4.7 \pm 7.1$ \\
\hline Duration $\geq \mathrm{I} h$ and/or DWI abnormality $(\mathrm{n})$ & $123(72.4 \%)$ \\
\hline Vertebrobasilar TIA (n) & $42(23.9 \%)$ \\
\hline ECD: stenoocclusion $(n)$ & $34(19.3 \%)$ \\
\hline TCD: abnormal (n) & $20(12.3 \%)$ \\
\hline
\end{tabular}

*Mean \pm standard deviation. 
ECD detected plaques without stenosis in $84(47.7 \%)$ patients and $\geq 50 \%$ stenosis or occlusions in $34(19.3 \%)$ patients. $24(13.6 \%)$ patients showed a stenosis of the cICA, $3(1.7 \%)$ an occlusion of the cICA, $4(2.3 \%)$ a stenosis of the cVA, $2(1.1 \%)$ an occlusion of the cVA, and 1 $(0.6 \%)$ stenosis or occlusions of both the cICA and cVA. 6 (3.4\%) patients had a high-grade cICA stenosis of $\geq 80 \%$. 5 of these patients underwent subsequent carotid endarterectomy and 1 patient stent-supported angioplasty.

TCD detected intracranial stenosis in $15(9.2 \%)$ patients and reactive collateral blood flow due to stenosis of the cICA in $5(3.1 \%)$ patients. $9(5.1 \%)$ patients showed a stenosis of the dICA or MCA, $1(0.6 \%)$ an occlusion of the dICA or MCA, $3(1.7 \%)$ a stenosis of the PCA, $1(0.6 \%)$ a stenosis of the VBA, and $1(0.6 \%)$ stenosis of both the cICA or MCA and PCA. In $13(7.4 \%)$ patients, TCD could not be applied because of inadequate temporal bone windows.

Tandem lesions of both the CICA and ipsilateral dICA or MCA were detectable in $3(1.8 \%)$ patients.

\section{Follow-up endpoints}

Follow-up data was available of $173(98.3 \%)$ patients. 9 $(5.7 \%)$ patients suffered an IS and $14(8.8 \%)$ a further TIA; 9 (5.7\%) more patients reported symptoms possibly consistent with cerebral ischemia but did not seek medical aid or had competing differential diagnoses as reported by the attending physician. In 7 of 14 patients with follow-up TIA, a new MRI was performed, which detected an acute ischemic lesion in only 1 patient.
$3(1.8 \%)$ patients experienced a MI and $2(1.2 \%)$ an ACS during follow-up; a further $4(2.4 \%)$ patients underwent surgical or endovascular revascularization in CAD, and 1 $(0.6 \%)$ patient had bypass surgery in PAD. Additionally, $4(2.4 \%)$ patients suffered from their first-ever angina pectoris attack, and $10(6.0 \%)$ patients experienced other vascular events (cardiac syncope, 4 cases; pacemaker implantation, 2 cases; aortic valve surgery, 1 case; WolffParkinson-White syndrome, 1 case; deep vein thrombosis, 1 case; pulmonary embolism, 1 case). 15 (8.5\%) patients died due to the following reasons: cardiac failure, 3 $(1.7 \%)$ cases; malignancy, $3(1.7 \%)$ cases; pneumonia, 2 $(1.1 \%)$ cases; unknown cause, $7(4.0 \%)$ cases.

Figure 1 shows the graded risk of new cerebral ischemic events and cardiovascular ischemic events based on ECD and TCD findings.

\section{Predictors of new cerebral ischemic events}

In univariate analysis (Table 2), detection of stenosis by ECD (hazard ratio (HR) $4.39,95 \%$ CI 1.93 to $9.99, P<$ 0.01 ), evidence of reactive collateral flow patterns or intracranial stenosis by TCD (HR 4.99, 95\% CI 1.97 to 12.62, $P<0.01$ ), and PAD (HR 7.64, 95\% CI 2.96 to $19.71, P<0.01)$ were significantly associated with followup IS or TIA. A trend that did not reach significance was also found for the parameters prolonged symptom duration (HR $1.04,95 \%$ CI 1.00 to $1.10, P=0.07$ ), advanced age (HR $1.03,95 \%$ CI 0.99 to $1.06, P=0.11$ ), and cardiac failure (HR 3.39, 95\% CI 1.00 to $11.55, P=0.05$ ). Evidence of acute ischemia on DWI had no significant effect in our study. Cox proportional hazards multivariate anal-

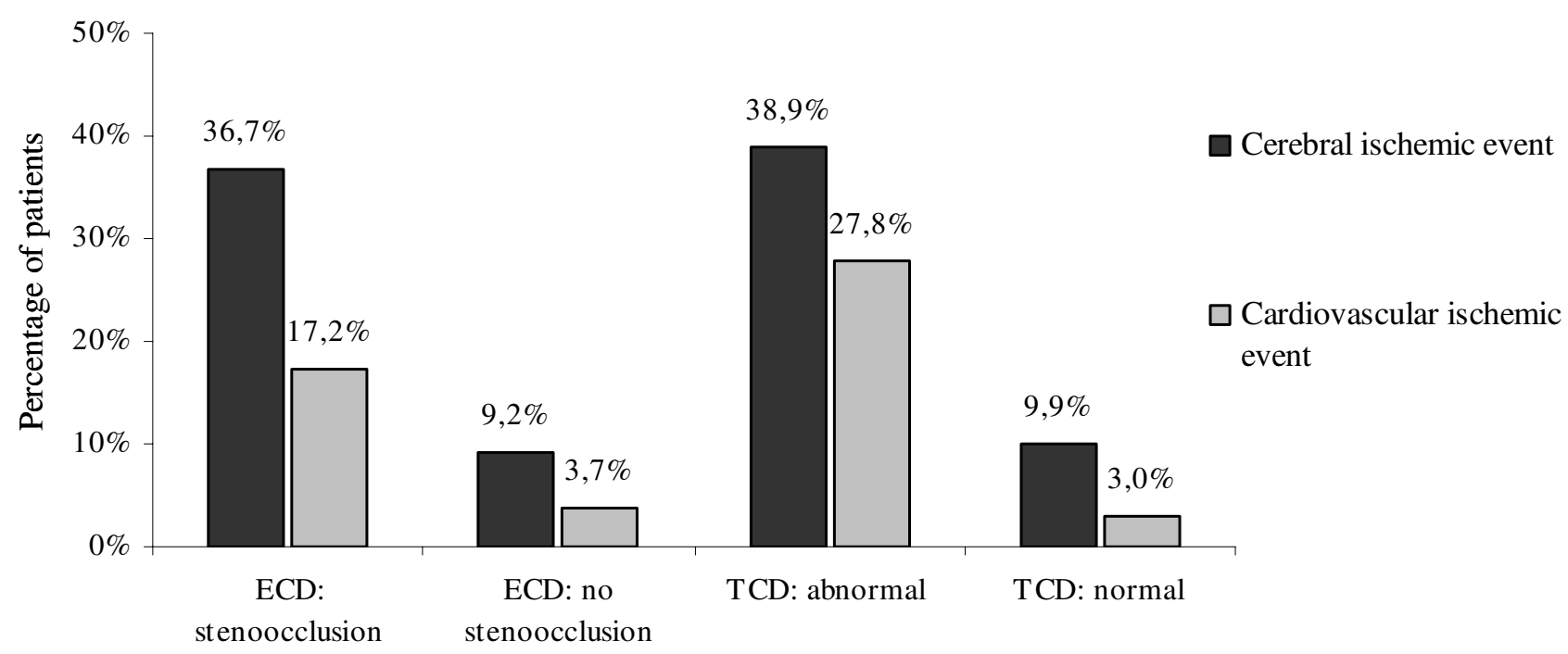

Figure I

Graded risk of new vascular events based on ECD and TCD findings. 
Table 2: Univariate analysis of variables possibly associated with new vascular events

\begin{tabular}{|c|c|c|c|c|c|c|}
\hline & \multicolumn{2}{|c|}{ Cerebral ischemic event } & \multicolumn{2}{|c|}{ Cardiovascular ischemic event } & \multicolumn{2}{|c|}{$\begin{array}{c}\text { Cerebral/cardiovascular ischemic event or vascular/ } \\
\text { unknown death }\end{array}$} \\
\hline & HR & $95 \% \mathrm{Cl}$ & HR & $95 \% \mathrm{Cl}$ & $\mathrm{HR}$ & $95 \% \mathrm{Cl}$ \\
\hline Age & 1.03 & 0.991 .06 & 1.04 & 0.991 .10 & 1.04 & 1.011 .07 \\
\hline Sex, female & 1.13 & 0.492 .62 & 0.93 & 0.233 .76 & 1.18 & 0.562 .45 \\
\hline Hypertension & 0.99 & 0.392 .51 & -* & $-*$ & 1.20 & 0.542 .68 \\
\hline Diabetes & 2.11 & 0.835 .35 & 5.00 & 1.4017 .86 & 1.85 & 0.824 .17 \\
\hline Hyperlipidemia & 0.57 & 0.241 .34 & 2.51 & 0.659 .70 & 0.51 & 0.241 .10 \\
\hline Body mass index $>30$ & 0.30 & 0.042 .24 & 1.06 & 0.215 .26 & 0.52 & 0.151 .76 \\
\hline Nicotine abuse & 1.30 & 0.572 .94 & 0.99 & 0.273 .69 & 1.06 & 0.532 .12 \\
\hline Atrial fibrillation & 0.94 & 0.283 .17 & -* & $-*$ & 1.02 & 0.352 .93 \\
\hline Coronary artery disease & 0.59 & 0.181 .99 & 2.27 & 0.628 .24 & 0.96 & 0.412 .24 \\
\hline Cardiac failure & 3.39 & 1.0011 .55 & 2.77 & 0.3422 .63 & 3.97 & 1.5110 .45 \\
\hline Peripheral artery disease & 7.64 & 2.9619 .71 & $-*$ & $-*$ & 7.42 & 3.2516 .94 \\
\hline DWI abnormality & 1.23 & 0.512 .93 & 0.59 & 0.122 .85 & 0.84 & 0.371 .90 \\
\hline Duration & 1.04 & 1.001 .10 & 0.92 & 0.791 .08 & 1.03 & 0.991 .08 \\
\hline $\begin{array}{l}\text { Duration } \geq \mathrm{I} h \text { and/or DWI } \\
\text { abnormality }\end{array}$ & 0.76 & 0.311 .89 & 0.69 & 0.172 .76 & 0.91 & 0.402 .07 \\
\hline Vertebrobasilar TIA & 0.17 & 0.021 .24 & 1.33 & 0.325 .46 & 0.39 & 0.121 .30 \\
\hline ECD: stenoocclusion & 4.39 & 1.939 .99 & 3.73 & $|.05| 3.3 \mid$ & 4.18 & 2.048 .59 \\
\hline TCD: abnormal & 4.99 & 1.9712 .62 & 9.62 & 2.4637 .68 & 5.13 & 2.2611 .67 \\
\hline
\end{tabular}

* Statistical analysis not possible due to small patient numbers.

SD: Standard deviation. HR: Hazard ratio. $\mathrm{Cl}$ : Confidence interval.

ysis (Table 3) confirmed pathological ECD and TCD findings (ECD: HR 4.30, 95\% CI 1.75 to $10.57, P=0.01$; TCD: HR $4.73,95 \%$ CI 1.86 to $12.04, P=0.01$ ) to be predictors of new cerebral ischemic events.

\section{Predictors of new cardiovascular ischemic events}

Detection of extracranial stenosis by ECD (HR 3.73, 95\% CI 1.05 to $13.31, P=0.04)$, proof of reactive collateral flow patterns or intracranial stenosis by TCD (HR 9.62, 95\% CI 2.46 to $37.68, P<0.01)$, and diabetes mellitus (HR 5.00, 95\% CI 1.40 to $17.86, P=0.01$ ) were significantly associated with the occurrence of MI, ACS, or revascularization procedures in univariate analysis (Table 2). However, whereas abnormal TCD findings (HR 18.51, $95 \%$ CI 3.49 to $98.24, P=0.001$ ) proved to predict cardiovascular ischemic events in multivariate analysis (Table 3), pathological ECD findings (HR 2.93, 95\% CI 0.77 to
$11.17, P=0.116)$ failed to reach significance in multivariate analysis.

\section{Predictors of the combined endpoint of cerebral ischemic events, cardiac ischemic events, and death of vascular or unknown cause}

In univariate analysis, detection of extracranial stenosis by ECD (HR 4.18, 95\% CI 2.04 to 8.59, $P<0.01$ ), evidence of reactive collateral flow patterns or intracranial stenosis by TCD (HR 5.13, 95\% CI 2.26 to $11.67, P<0.01$ ), advanced age (HR 1.04, 95\% CI 1.01 to $1.07, P<0.01$ ), PAD (HR 7.42, 95\% CI 3.25 to $16.94, P<0.01$ ), and cardiac failure (HR 3.97, 95\% CI 1.51 to $10.45, P<0.01$ ) were significantly associated with the combined endpoint of IS or TIA, MI or ACS, and death of vascular or unknown cause. Both pathological ECD and TCD findings (ECD: HR 3.46, 95\% CI 1.56 to $7.66, P=0.02$; TCD: HR 4.97,

Table 3: Predictors of new vascular events in multivariate analysis*

\begin{tabular}{|c|c|c|c|c|c|c|}
\hline & \multicolumn{2}{|c|}{ Cerebral ischemic event } & \multicolumn{2}{|c|}{ Cardiovascular ischemic event } & \multicolumn{2}{|c|}{$\begin{array}{c}\text { Cerebral/cardiovascular ischemic event or vascular/unknown } \\
\text { death }\end{array}$} \\
\hline & $H R$ & $95 \% \mathrm{Cl}$ & $\mathrm{HR}$ & $95 \% \mathrm{Cl}$ & HR & $95 \% \mathrm{Cl}$ \\
\hline ECD: stenoocclusion & 4.30 & 1.7510 .57 & 2.93 & 0.7711 .17 & 3.46 & 1.567 .66 \\
\hline TCD: abnormal & 4.73 & 1.8612 .04 & $|8.5|$ & 3.4998 .24 & 4.97 & 2.1611 .47 \\
\hline
\end{tabular}

* Cox proportional hazards multivariate analysis adjusted for age and sex.

HR: Hazard ratio. Cl: Confidence interval. 
95\% CI 2.16 to $11.47, P<0.001)$ proved to be predictors of the combined endpoint in multivariate analysis (Table $3)$.

\section{Discussion}

The present study demonstrates that TIA patients with ultrasonographic evidence of extracranial or intracranial stenoocclusive disease are at high risk of further cerebral ischemic events during medium- to long-term follow-up. After a median follow-up of 27 months, nearly $40 \%$ of the patients with either stenoocclusive disease in ECD or pathological findings in TCD have suffered a new IS or TIA. Several studies have already reported an increased stroke incidence after TIA of large-artery atherosclerotic or cardioembolic etiology as compared to other subtypes, but to our knowledge only for short- and medium-term follow-up [3,13-15]. Purroy et al., in this context, have shown a 3-month stroke risk of $20 \%$ after TIA due to largeartery atherosclerosis [14]. The present data demonstrate that detection of stenoocclusive disease by ECD or TCD is not only associated with a higher short-term stroke risk after TIA but remains a predictor of recurrent cerebral ischemia during medium- to long-term follow-up.

Whereas several previous studies found a significant association between prolonged symptom duration and recurrent cerebral ischemic events during short- and mediumterm follow-up after TIA $[2,8-10,13]$, there was only a trend that did not reach significance in this study. We also could not show that TIA patients with acute ischemia on DWI are at higher risk of further cerebral ischemic events on medium- to long-term follow-up. Because of the limited number of patients, however, these findings may be simply explained by chance.

As an additional result of the present study, vertebrobasilar TIA allocation seems to be associated with a lower recurrence rate of cerebral ischemia. This finding is consistent with a recent systematic review of Flossmann et al., who noted a lower stroke incidence in patients with vertebrobasilar TIA or minor stroke when data was confined to hospital-based studies (OR 0.68, 95\% CI 0.6 to 0.8 ), but a higher stroke incidence when data was restricted to population-based studies (OR 1.48,95\% CI 1.1 to 2.0) [33].

A second major finding of the present study is that detection of reactive collateral flow patterns or intracranial stenosis by TCD predicts new cardiovascular ischemic events on medium- to long-term follow-up after TIA. 5 of 18 $(27.8 \%)$ patients with abnormal TCD findings, but only 4 of $134(3 \%)$ patients without, have developed a subsequent cardiovascular ischemic event. The association between TCD findings and cardiovascular prognosis is of particular importance as cardiovascular disease becomes the major cause of death on long-term follow-up after TIA [22].
A high prevalence of asymptomatic CAD in patients with cerebrovascular disease is well known [27]. Chimowitz et al. documented abnormal cardiac stress tests in 50\% of TIA or stroke patients with large-artery occlusive disease [34], with the rates being $25 \%$ in isolated intracranial artery stenosis, $50 \%$ in isolated extracranial carotid stenosis, and even $83 \%$ in coexistent extracranial carotid and intracranial artery stenosis [34]. In another study, symptomatic intracranial atherosclerosis was associated with a $52 \%$ risk of occult CAD [35]. However, whereas a strong correlation between the extent of extracranial carotid and coronary atherosclerosis is well accepted in Caucasians [36], and guidelines already recommend coronary risk evaluation in TIA patients based on the individual cardiovascular risk profile and the prevalence of carotid artery disease [27], the relationship between intracranial atherosclerosis and CAD has not been sufficiently evaluated in this ethnic group. In Asians, who suffer from intracranial atherosclerosis more frequently than Caucasians, correlation between extracranial carotid and coronary atherosclerosis seems to be stronger than between intracranial artery and coronary atherosclerosis [37].

Whereas pathological TCD findings proved to be a predictor of new cardiovascular ischemic events in the present study, detection of stenoocclusive disease by ECD failed significance in multivariate analysis. However, as our definition of stenoocclusive disease in ECD included both cICA and cVA lesions, the results cannot be equated with the prognostic value of isolated extracranial carotid disease, which might be higher. Moreover, the definition of pathological TCD findings referred to both reactive collateral blood flow secondary to extracranial lesions and intracranial stenoocclusive disease in this study, thus probably characterizing those TIA patients with the highest risk of generalized atherosclerosis and consequently cardiovascular ischemic events. TCD is already accepted as an accurate, safe, and cost-effective diagnostic tool for the detection of intracranial stenoocclusive disease $[38,39]$, and is widely accessible in most countries. The results of the present study support the routine use of TCD in addition to ECD in TIA patients. Moreover, routine screening tests for CAD and aggressive prevention therapies should be considered in TIA patients with pathological TCD findings.

The present study has several limitations. A larger patient cohort would have been necessary to improve the statistical power of the study and allow further subgroup analyses. Moreover, follow-up was conducted as telephone or mail interview only. Even though attending physicians and/or hospitals were contacted to complete data, early and minor vascular events may have been missed due to limited ability of the patients to recall their symptoms over the complete follow-up period. A further weakness of the present study is the lack of angiographic validation of 
ECD and TCD findings. However, both ECD and TCD have been shown to be accurate diagnostic tools for the detection of extracranial and intracranial stenoocclusive disease [38-40]. Finally, ECD and TCD classification did not distinguish between symptomatic and asymptomatic vessel disease in the present study.

\section{Conclusion}

The present study confirms the high risk of further cerebral ischemic events in TIA patients with ultrasonographic evidence of extracranial or intracranial stenoocclusive disease during medium- to long-term follow-up. As pathological TCD findings additionally predict new cardiovascular ischemic events, routine screening tests for $\mathrm{CAD}$ and aggressive prevention therapies should be considered in this subgroup of TIA patients. ECD and TCD are important diagnostic procedures in patients with TIA.

\section{Competing interests}

The authors declare that they have no competing interests.

\section{Authors' contributions}

KH carried out the data collection and drafted the manuscript. SS participated in its design and data collection. LE participated in the follow-up data collection and has been involved in drafting the manuscript. $\mathrm{AB}$ performed the statistical analyses. DS revised the manuscript critically for important intellectual content and helped to draft the manuscript. BH made substantial contributions to conception, revised the manuscript and gave final approval of the version to be published. HP conceived of the study, and participated in its design and coordination and helped to draft the manuscript. All authors read and approved the final manuscript.

\section{Additional material}

\section{Additional file 1}

Baseline characteristics of patients with and without abnormal TCD. The data provided represent the baseline characteristics of the patients with and without abnormal TCD.

Click here for file

[http://www.biomedcentral.com/content/supplementary/14712342-9-13-S1.doc]

\section{Acknowledgements}

We would like to thank Suzann Pilotto, Beate Eckenweber, Claudia Leege, Christina Leonhart, and Romy Siegert for their contributions to this study.

\section{References}

I. Hill MD, Yiannakoulias N, Jeerakathil T, Tu JV, Svenson LW, Schopflocher DP: The high risk of stroke immediately after transient ischemic attack: a population-based study. Neurology 2004, 62(II):2015-2020.
2. Johnston SC, Gress DR, Browner WS, Sidney S: Short-term prognosis after emergency department diagnosis of TIA. Jama 2000, 284(22):290 I-2906.

3. Eliasziw M, Kennedy J, Hill MD, Buchan AM, Barnett HJ: Early risk of stroke after a transient ischemic attack in patients with internal carotid artery disease. Cmaj 2004, I 70(7): I I05-I I09.

4. Kleindorfer D, Panagos P, Pancioli A, Khoury J, Kissela B, Woo D, Schneider A, Alwell K, Jauch E, Miller R, Moomaw C, Shukla R, Broderick JP: Incidence and short-term prognosis of transient ischemic attack in a population-based study. Stroke; a journal of cerebral circulation 2005, 36(4):720-723.

5. Lisabeth LD, Ireland JK, Risser JM, Brown DL, Smith MA, Garcia NM, Morgenstern LB: Stroke risk after transient ischemic attack in a population-based setting. Stroke; a journal of cerebral circulation 2004, 35(8): $1842-1846$.

6. Coutts SB, Simon JE, Eliasziw M, Sohn CH, Hill MD, Barber PA, Palumbo V, Kennedy J, Roy J, Gagnon A, Scott JN, Buchan AM, Demchuk AM: Triaging transient ischemic attack and minor stroke patients using acute magnetic resonance imaging. Annals of neurology 2005, 57(6):848-854.

7. Rao SV, Ohman EM, Granger CB, Armstrong PW, Gibler WB, Christenson RH, Hasselblad V, Stebbins A, McNulty S, Newby LK: Prognostic value of isolated troponin elevation across the spectrum of chest pain syndromes. Am J Cardiol 2003, 9I(8):936-940.

8. Correia M, Silva MR, Magalhaes R, Guimaraes L, Silva MC: Transient ischemic attacks in rural and urban northern Portugal: incidence and short-term prognosis. Stroke; a journal of cerebral circulation 2006, 37(I):50-55.

9. Johnston SC, Rothwell PM, Nguyen-Huynh MN, Giles MF, Elkins JS, Bernstein AL, Sidney S: Validation and refinement of scores to predict very early stroke risk after transient ischaemic attack. Lancet 2007, 369(9558):283-292.

10. Rothwell PM, Giles MF, Flossmann E, Lovelock CE, Redgrave JN, Warlow CP, Mehta Z: A simple score (ABCD) to identify individuals at high early risk of stroke after transient ischaemic attack. Lancet 2005, 366(9479):29-36.

II. Prabhakaran S, Chong JY, Sacco RL: Impact of abnormal diffusion-weighted imaging results on short-term outcome following transient ischemic attack. Arch Neurol 2007, 64(8): 11 105-1109.

12. Douglas VC, Johnston CM, Elkins J, Sidney S, Gress DR, Johnston SC: Head computed tomography findings predict short-term stroke risk after transient ischemic attack. Stroke; a journal of cerebral circulation 2003, 34( ( 2):2894-2898.

13. Purroy F, Montaner J, Rovira A, Delgado P, Quintana M, Alvarez-Sabin $\mathrm{J}$ : Higher risk of further vascular events among transient ischemic attack patients with diffusion-weighted imaging acute ischemic lesions. Stroke; a journal of cerebral circulation 2004, 35(10):2313-2319.

14. Purroy F, Montaner J, Molina CA, Delgado P, Ribo M, Alvarez-Sabin J: Patterns and predictors of early risk of recurrence after transient ischemic attack with respect to etiologic subtypes. Stroke; a journal of cerebral circulation 2007, 38(I 2):3225-3229.

15. Lin HJ, Yeh PS, Tsai TC, Cheng TJ, Ke D, Lin KC, Ho JG, Chang CY: Differential risks of subsequent vascular events for transient ischaemic attack and minor ischaemic stroke. J Clin Neurosci 2007, I 4(I): I7-2I.

16. Tsivgoulis G, Spengos K, Manta P, Karandreas N, Zambelis T, Zakopoulos $N$, Vassilopoulos $D$ : Validation of the $A B C D$ score in identifying individuals at high early risk of stroke after a transient ischemic attack: a hospital-based case series study. Stroke; a journal of cerebral circulation 2006, 37( I 2):2892-2897.

17. Tsivgoulis G, Vassilopoulou S, Spengos K: Potential applicability of ABCD score in triaging TIA patients. Lancet 2007, 369(9567): 1082.

18. Josephson SA, Sidney S, Pham TN, Bernstein AL, Johnston SC: Higher ABCD2 score predicts patients most likely to have true transient ischemic attack. Stroke; a journal of cerebral circulation 2008, 39( I I ):3096-3098.

19. Hankey GJ, Slattery JM, Warlow CP: The prognosis of hospitalreferred transient ischaemic attacks. Journal of neurology, neurosurgery, and psychiatry I99I, 54(9):793-802.

20. Dennis M, Bamford J, Sandercock P, Warlow C: Prognosis of transient ischemic attacks in the Oxfordshire Community 
Stroke Project. Stroke; a journal of cerebral circulation 1990 , 2 I (6):848-853.

21. Carolei A, L C, M F, BM F, Motolese MFC: Long-term prognosis of transient ischemic attcks and reversible ischemic neurologic deficts: a hospital-based study. Cerebrovasc Dis 1992, 2(5):266-272.

22. Hankey G]: Long-term outcome after ischaemic stroke/transient ischaemic attack. Cerebrovasc Dis 2003, 16(Suppl I): I4-19.

23. van Wijk I, Kappelle LJ, van Gijn J, Koudstaal PJ, Franke CL, Vermeulen M, Gorter JW, Algra A: Long-term survival and vascular event risk after transient ischaemic attack or minor ischaemic stroke: a cohort study. Lancet 2005, 365(9477):2098-2 104.

24. Di Pasquale G, Andreoli A, Pinelli G, Grazi P, Manini G, Tognetti F, Testa C: Cerebral ischemia and asymptomatic coronary artery disease: a prospective study of 83 patients. Stroke; a journal of cerebral circulation 1986, I7(6): I098-I I0I.

25. Rokey R, Rolak LA, Harati Y, Kutka N, Verani MS: Coronary artery disease in patients with cerebrovascular disease: a prospective study. Annals of neurology 1984, I6(1):50-53.

26. Love BB, Grover-McKay M, Biller J, Rezai K, McKay CR: Coronary artery disease and cardiac events with asymptomatic and symptomatic cerebrovascular disease. Stroke; a journal of cerebral circulation 1992, 23(7):939-945.

27. Adams RJ, Chimowitz MI, Alpert JS, Awad IA, Cerqueria MD, Fayad $P$, Taubert KA: Coronary risk evaluation in patients with transient ischemic attack and ischemic stroke: a scientific statement for healthcare professionals from the Stroke Council and the Council on Clinical Cardiology of the American Heart Association/American Stroke Association. Stroke; a journal of cerebral circulation 2003, 34(9):2310-2322.

28. Special report from the National Institute of Neurological Disorders and Stroke. Classification of cerebrovascular diseases III. Stroke; a journal of cerebral circulation 1990, 21(4):637-676.

29. Hankey G], Slattery JM, Warlow CP: Transient ischaemic attacks: which patients are at high (and low) risk of serious vascular events? J Neurol Neurosurg Psychiatry 1992, 55(8):640-652.

30. Mead GE, Lewis SC, Wardlaw JM, Dennis MS: Comparison of risk factors in patients with transient and prolonged eye and brain ischemic syndromes. Stroke; a journal of cerebral circulation 2002, 33(I0):2383-2390.

3I. Baumgartner RW, Mattle HP, Schroth G: Assessment of $>I=50 \%$ and $<\mathbf{5 0} \%$ intracranial stenoses by transcranial color-coded duplex sonography. Stroke; a journal of cerebral circulation 1999, 30(I):87-92.

32. Wong KS, Li H, Chan YL, Ahuja A, Lam WW, Wong A, Kay R: Use of transcranial Doppler ultrasound to predict outcome in patients with intracranial large-artery occlusive disease. Stroke; a journal of cerebral circulation 2000, 3 I (I I):264 I-2647.

33. Flossmann E, Rothwell PM: Prognosis of vertebrobasilar transient ischaemic attack and minor stroke. Brain 2003, 126(Pt 9): 1940-1954.

34. Chimowitz MI, Poole RM, Starling MR, Schwaiger M, Gross MD: Frequency and severity of asymptomatic coronary disease in patients with different causes of stroke. Stroke; a journal of cerebral circulation 1997, 28(5):941-945.

35. Arenillas JF, Candell-Riera J, Romero-Farina G, Molina CA, Chacon P, Aguade-Bruix S, Montaner J, de Leon G, Castell-Conesa J, AlvarezSabin J: Silent myocardial ischemia in patients with symptomatic intracranial atherosclerosis: associated factors. Stroke; a journal of cerebral circulation 2005, 36(6): I20I-I 206.

36. Craven TE, Ryu JE, Espeland MA, Kahl FR, McKinney WM, Toole JF, McMahan MR, Thompson CJ, Heiss G, Crouse JR 3rd: Evaluation of the associations between carotid artery atherosclerosis and coronary artery stenosis. A case-control study. Circulation 1990, 82(4): I230-I242.

37. Bae HJ, Yoon BW, Kang DW, Koo JS, Lee SH, Kim KB, Lee J, Roh JK: Correlation of coronary and cerebral atherosclerosis: difference between extracranial and intracranial arteries. Cerebrovasc Dis 2006, 2 I ( I 2): I I 2-1 I 9.

38. Navarro JC, Lao AY, Sharma VK, Tsivgoulis G, Alexandrov AV: The accuracy of transcranial Doppler in the diagnosis of middle cerebral artery stenosis. Cerebrovasc Dis 2007, 23(56):325-330.

39. Tsivgoulis G, Sharma VK, Lao AY, Malkoff MD, Alexandrov AV: Validation of transcranial Doppler with computed tomography angiography in acute cerebral ischemia. Stroke; a journal of cerebral circulation 2007, 38(4): I245-1249.
40. Carriero A, Ucchino S, Magarelli N, Legnini M, Macri MA, Napolitano AM, Bonomo L: Carotid bifurcation stenosis: a comparative study between MR angiography and duplex scanning with respect to digital subtraction angiography. J Neuroradiol 1995, 22(2): | $03-1 \mid I$.

\section{Pre-publication history}

The pre-publication history for this paper can be accessed here:

http://www.biomedcentral.com/1471-2342/9/13/prepub
Publish with Biomed Central and every scientist can read your work free of charge

"BioMed Central will be the most significant development for disseminating the results of biomedical research in our lifetime. "

Sir Paul Nurse, Cancer Research UK

Your research papers will be:

- available free of charge to the entire biomedical community

- peer reviewed and published immediately upon acceptance

- cited in PubMed and archived on PubMed Central

- yours - you keep the copyright

Submit your manuscript here:

http://www.biomedcentral.com/info/publishing_adv.asp
BioMedcentral 